\title{
APROVEITAMENTO DE CONDENSADO E UTILIZAÇÃO DE VAPOR FLASH NO EXPANDER
}

\author{
Eduardo Rasi de Almeida Prado \\ Flávio Luis Lemos ${ }^{1}$ \\ Alessandro Trombeta ${ }^{1}$ \\ Bruno Raphael Ferraz de Almeida ${ }^{2}$
}

\begin{abstract}
Resumo: Numa indústria de extração de óleo de soja, há diversas etapas desde que o grão sai do campo até a obtençáo do óleo refinado, pronto para o consumo humano. Dentre essas etapas, a preparação dos grãos apresenta grande importância no processo, pois representa potencial para se obter significativo aumento no rendimento da extração. Ainda, a preparação dos grãos apresenta duas subetapas de grande importância, sendo elas o condicionamento dos grãos e a expansão da massa. Estas subetapas ocorrem no interior do condicionador e no interior do expander, respectivamente. No condicionador ocorre o pré-cozimento dos grãos por injeçáo indireta de vapor. Já a expansão ocorre por meio do contato direto com vapor a pressóes mais altas que a atmosférica, o que ocasiona diversas mudanças na massa que vai sofrer o processo de extração. Neste trabalho foi realizado um estudo sobre a redução do consumo de vapor utilizado no expander, utilizando-se parcialmente o condensado proveniente do condicionador vertical. Este condensado passou por um tanque de flash e o vapor formado foi direcionado ao expander, diminuindo assim a quantidade utilizada de vapor proveniente da caldeira.
\end{abstract}

Palavras-chaves: Expander, vapor flash, recuperação de condensado.

Abstract: In an industry of extraction of soybean oil, there are several steps since the grain leaves the field to obtain the refined oil, ready for human consumption. Among these steps, the preparing of the grains has great importance in the process because it represents potential to achieve significant increase in the yield of extraction. Still, the preparation of grain has two sub-steps of great importance. They are the conditioning and the expansion of the grain mass. These sub-steps take place inside the conditioner and the interior of the expander, respectively. In the conditioner occurs the pre-cooking of the beans through indirect injection of steam. In the expander occurs the expansion of the grains through direct contact with steam at pressures higher than atmospheric pressure, which causes several changes in the mass that will suffer the extraction process. In this work we present a study on reducing the consumption of steam used in the expander, using partially the condensate from the vertical conditioner. This condensate passed through a flash tank and the steam formed was directed to expand, thereby decreasing the amount used steam from the boiler.

Keywords: Expander, flash steam, condensate recovery.

\footnotetext{
${ }^{1}$ Programa de pós-graduação em Engenharia Química da Universidade Estadual de Maringá - UEM. Avenida Colombo, 5790 - Bloco E46. CEP 87020-900. Email: dudarasi@yahoo.com.br; flavioluislemos@yahoo.com.br; alessandrotrombeta@yahoo. com.br.

${ }^{2}$ Graduação em Engenharia Química na Universidade Estadual de Maringá - UEM. Avenida Colombo, 5790 - Bloco D90. CEP 87020-900. Email: bruno_ferraz2@hotmail.com
} 


\section{INTRODUÇÃO}

O crescimento da demanda por produtos com maior valor agregado e a oportunidade de obtenção de maior volume de divisas com a exportação desses produtos exigiu grandes modificaçóes na estrutura, no tamanho e nos processos de produção das empresas de processamento de soja no Brasil. Essas modificaçóes visaram atender à demanda crescente, a melhoria de rendimento e, principalmente, a redução do consumo de energia, a qual está ligada diretamente à redução de custos (Paraíso, 1984).

A industrialização de oleaginosas constitui-se num dos mais importantes setores do sistema agroindustrial, pela importância de seus produtos nas indústrias de cosméticos e como matéria-prima no processamento de alimentos para o consumo animal e humano (Barbosa, 1998).

O óleo de soja corresponde a mais de $90 \%$ da produção de óleos vegetais. A soja, portanto, constitui a mais importante matéria-prima da cadeia de produção de óleos vegetais no Brasil. No período 2004/2005 a produção de soja no Brasil correspondeu a $23,7 \%$ da produção mundial e a exportação do complexo soja correspondeu a $31,5 \%$ no farelo e $26,2 \%$ no óleo, correspondendo ao segundo maior exportador mundial (ABIOVE).

Nesse contexto, a procura por métodos mais rápidos e eficientes de fabricação é de grande importância no mercado. Ainda, o rendimento da extração do óleo de soja é grandemente influenciado pelo método de preparação da massa a ser introduzida no extrator. Para tanto, o vapor apresenta grande utilidade, pois serve para condicionar e expandir a massa. Estas etapas serão detalhadas mais a frente.

\section{OBJETIVOS}

Redução no consumo de vapor utilizado no expander, analisando-se um sistema de recolhimento de condensado do condicionador e formaçáo de vapor flash, no qual este vapor formado é injetado no expander.

\section{REVISÃO BIBLIOGRÁFICA}

\subsection{UTILIZAÇÃO DE VAPOR NA INDÚSTRIA}

O vapor apresenta diversas utilidades de vital importância na indústria, dentre elas o aquecimento de líquidos, cozimento de massas, preparação de grãos para etapas posteriores. Após ocorrer a troca de calor, ocorre a formação de condensado (Passolongo, 2008).

Ainda segundo o autor, o condensado, salvo nos casos de contaminação, constitui uma água de boa qualidade, previamente tratada, podendo ser reaproveitada na alimentaçáo da caldeira e, assim, economizar água de reposiçáo e respectivo tratamento

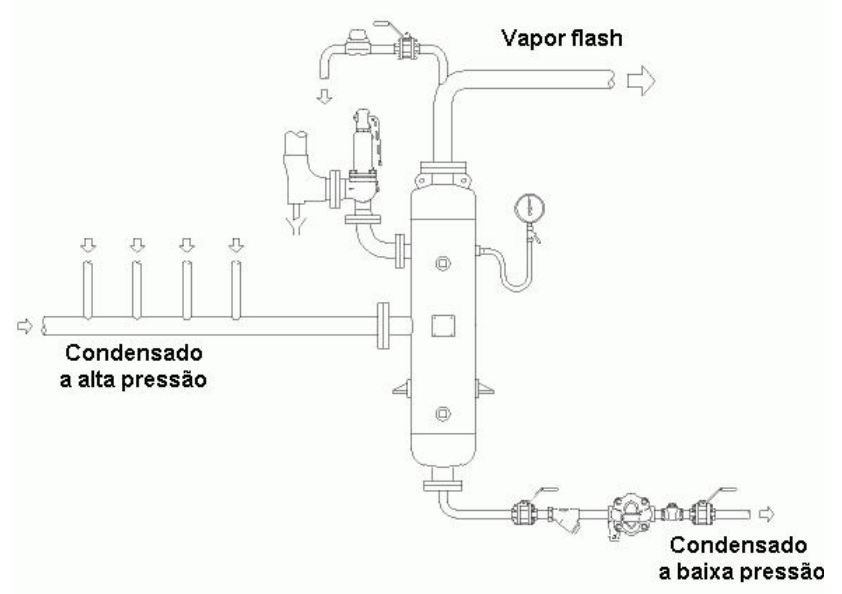

Figura 1: Esquema do funcionamento de um tanque flash.

Vapor saturado tem a grande vantagem de manter temperatura constante durante a condensação à pressão constante. A pressão de condensaçáo do vapor saturado controla indiretamente a temperatura dos processos. $\mathrm{O}$ controle de pressão, por ser um controle mecânico de ação direta é conseguido muito mais facilmente que o controle direto de temperatura (Bizzo, 2011).

\subsection{EXTRAÇÃO DO ÓLEO DE SOJA}

O processo de obtenção de óleo de soja bruto é uma parte importante na indústria de óleo de soja comestível e constitui-se das seguintes etapas: preparação dos grãos, extração e recuperação do solvente, como ilustrado pela figura abaixo. 


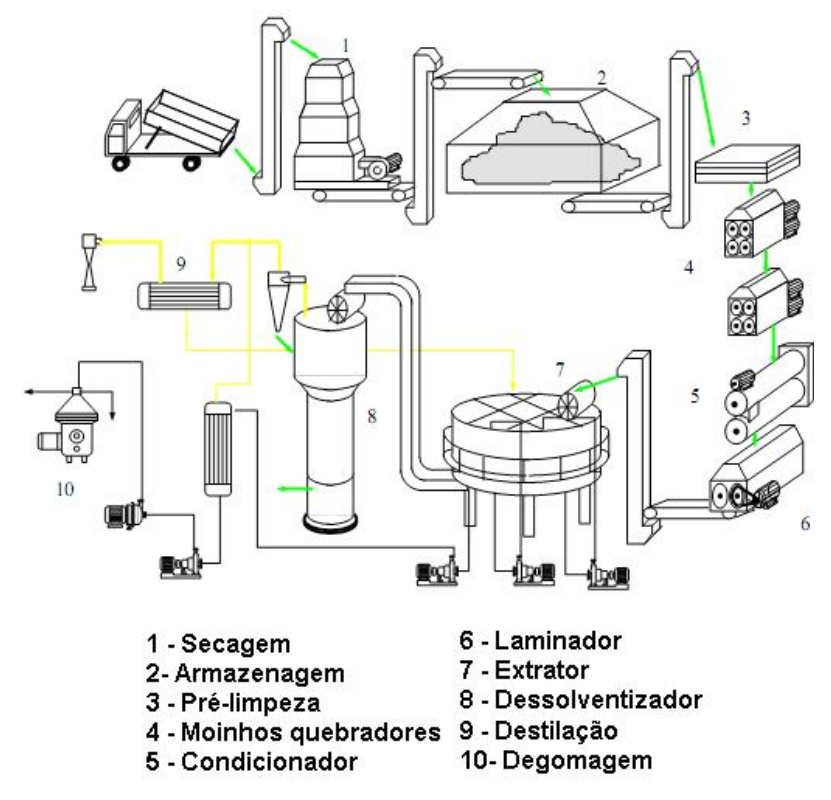

Figura 2: Fluxograma simplificado do processamento do grão de soja.

O processo de obtenção do óleo está originalmente relacionado com a extração de lipídios das sementes vegetais. Os métodos comumente empregados nesta obtenção são a prensagem, a extração por solvente ou a combinação destes, também chamado de misto (Savasini, 1981).

O autor também cita que o processo de extração por solvente é o mais amplamente utilizado e o mais eficiente em termos de consumo de energia e residual de óleo na torta. Este consome energia em torno de 20 a $25 \mathrm{kWh}$ de energia por tonelada de soja e gera um residual de óleo na torta em torno de $0,8 \%$. O método de prensagem usa, normalmente, prensas de alta pressáo, que são bastante flexíveis para operar com diferentes tipos de oleaginosas. Entretanto, este processo está totalmente ultrapassado, pois além de deixar um residual de óleo na torta muito alto ( 4 a $5 \%$ ) ele consome muita energia. Em média 65,0 kWh de energia são utilizados para cada tonelada de soja processada. Já o processo denominado misto utiliza cerca de 46,0 kWh de energia por tonelada de soja processada e o residual de óleo na torta é de 1,1 a $1,4 \%$. Portanto, o método de extração por solvente é o escolhido pela maioria das indústrias de extração de óleo.

O processo de extração do óleo de soja, desde a chegada dos grãos até a fase de envase apresenta várias etapas. Somente a preparação dos grãos será detalhada, pois nesta etapa foram implementadas as mudanças propostas.

\subsection{PREPARAÇÃO DOS GRÃOS}

A preparação é uma fase intermediária entre o armazenamento e a extraçáo do óleo de soja. Nesta etapa o grão é preparado para aumentar a capacidade de extraçáo, mas antes de serem processados, os grãos devem ser limpos e é nesta etapa que sujidades e são removidas por peneiras vibratórias sob ventilação, e impurezas metálicas são eliminadas por meio de imãs instalados próximo às peneiras (Amaral, Jaigobind e Jaigobind, 2006).

Os autores dizem ainda que esta etapa abrange os processos de quebra, separaçáo de cascas, condicionamento, laminação e expansão do grão de soja.

\subsubsection{Quebra dos gráos}

Tem por objetivo reduzir o tamanho do grão para que a matéria-prima possa ser laminada. Há um aumento na superfície de saída do óleo, pois a trituração diminui a distância entre o centro da semente e sua superfície e facilita o rompimento do tecido das paredes celulares. Porém, a desintegração ativa as enzimas celulares principalmente lipase e peroxidase, gerando um efeito negativo sobre a qualidade do óleo e da torta ou farelo de soja. Daí a necessidade de que este processo seja feito rapidamente e seguido de inativação das enzimas pelo cozimento (Amaral, Jaigobind e Jaigobind, 2006).

\subsubsection{Separação de cascas}

Nas colunas de ar ocorre a separaçáo entre a polpa e as cascas. A mistura de polpa e casca entra na coluna pela parte superior, enquanto ar passa de baixo para cima arrastando consigo as cascas, pois estas apresentam menor peso que a polpa (Thomas, 2003).

\subsubsection{Condicionamento}

No condicionador há a injeção de vapor indireto, visando aumentar a temperatura dos grãos. Este cozimento tem a finalidade de dar à matériaprima certa plasticidade, formando flocos de resistência mecânica apropriada. Este cozimento possibilita a formação de lâminas (Thomas, 2003).

\subsubsection{Laminaçáo}

A laminação é um processo onde o material sólido passa entre cilindros lisos que funcionam em rotaçóes diferentes e sentidos opostos que rompem e distorcem as células, formando as cha- 
madas lâminas (apresentam espessura em torno de $0,35 \mathrm{~mm}$ ). Esta etapa desempenha a função de aumentar a superfície de contato entre o sólido e o solvente. Resumindo, obtém-se um maior contato entre as fases com melhor penetração e drenagem de solvente no leito do extrator (Custódio, 2003).

$\mathrm{O}$ autor aponta ainda que os flocos quando muito finos, apresentam uma permeabilidade elevada, mas em contrapartida, produzem leitos com baixa percolabilidade. Da mesma forma, quando se tem flocos mais espessos, têm-se leitos com alta percolabilidade e menor permeabilidade. Por fim, a laminação entra para gerar um equilíbrio entre as fases do processo com boa permeabilidade no floco e percolabilidade no leito de extraçáo.

\subsubsection{Expansáo da massa laminada}

A última etapa de preparação dos grãos é a expansão. Esta expansão modifica as propriedades do material, aumentando a densidade e porosidade e, assim, facilitando o processo de extraçáo por solvente (Custódio, 2003). Este processo e o equipamento onde esta expansão ocorre serão descritos detalhadamente a seguir.

\subsection{EXPANDER}

Os expanders são usados para preparar a massa que será utilizada no extrator desde 1965. Apresentam design relativamente simples: são constituídos por um duto, no qual há uma rosca que serve para movimentar, direcionar e comprimir a massa que está sendo adicionada; além disso, apresentam entradas laterais por onde o vapor é injetado na massa. Por fim, há um componente denominado auto-cone, onde ocorre a expansão da massa (Lopes, 2008).

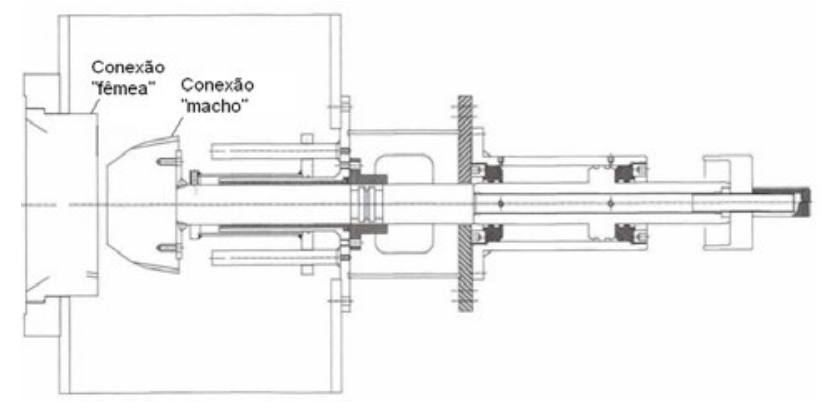

Figura 3: Auto-cone detalhado

Este auto-cone está conectado a um compressor e a pressão exercida pela conexão "macho" sobre a conexão "fêmea" é ajustável para obtenção de uma melhor massa expandida.

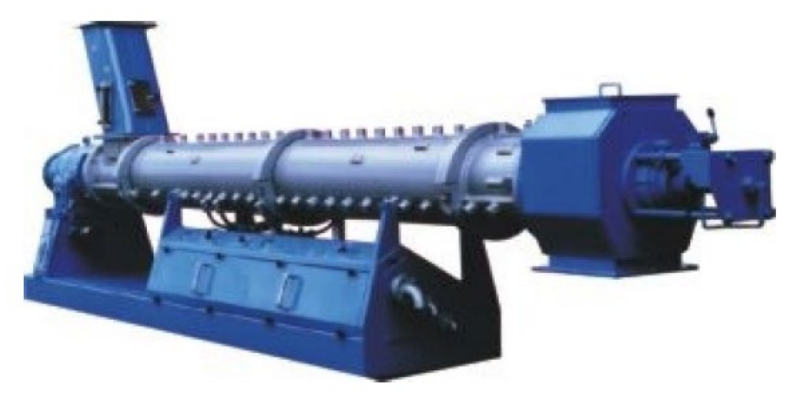

Figura 4: Expander

Estes aparelhos melhoram o rendimento dos extratores através do aumento da densidade dos flocos de massa. As partículas após as etapas de condicionamento e laminação são comprimidas a temperaturas bem superiores à do ponto de ebulição da água. Esta alta temperatura é obtida injetando-se vapor diretamente na massa. Lopes diz ainda que quando atinge a saída do expander (auto-cone), que está ao nível da pressão atmosférica, o vapor de água expande-se, modificando as propriedades do material em questão.

\subsubsection{Benefícios da utilizaçáo de massa expandida}

Segundo Ericksson (1995), a utilização de massa expandida apresenta diversos benefícios quando comparada à utilização de massa laminada. Dentre eles:

- acréscimo da densidade em relação ao material laminado $\left(300 \mathrm{~kg} / \mathrm{m}^{3}\right.$ da massa laminada para $550 \mathrm{Kg} / \mathrm{m}^{3} \mathrm{da}$ massa expandida), aumentando assim a área de contato sólido-líquido de 40 para $50 \mathrm{~m}^{2}{ }_{\text {massa }} / \mathrm{m}^{3}$ solvente;

- a massa expandida é mais porosa e não restringe a percolabilidade do solvente, possibilitando melhor drenagem do material sólido ao final da extração, resultando em uma remoção de óleo mais completa;

- menor arraste de solvente pelos sólidos e consequentemente menos consumo de energia durante a dessolventização;

- a pressão rompe as paredes celulares da soja, facilitando o contato entre o solvente e o óleo presente no interior das células.

\subsection{MEDIDA DO CONSUMO DE VAPOR}

Medidores do tipo Vortex - Estes medidores exploram o fenômeno conhecido como Ka- 
mann Vortex e são utilizados na medição de vazão de líquidos de baixa viscosidade, gases e vapor (saturado e superaquecido). Os medidores Vortex se caracterizam pela ausência de partes móveis em contato com o fluido, baixa perda de carga e boa exatidão (Delmée, 2003).

Ainda, segundo o autor, seu funcionamento é baseado na medição de velocidade do fluído a partir da quantidade de vórtices formados quando o líquido passa por um pequeno objeto estático que cruza o interior do tubo. Um sensor localizado após este objeto (que pode ser piezoelétrico ou ultra-sônico) monitora continuamente os vórtices gerados enviando um sinal que será processado por um circuito eletrônico microprocessado. Uma vez que se conhece a secçáo transversal do tubo e tendo-se o valor da velocidade, a vazão pode ser determinada.

\section{MATERIAIS E MÉTODOS}

\subsection{INSTALAÇÃO DO TANQUE DE FLASH}

Para que o condensado pudesse ser reaproveitado, teve-se que instalar um tanque de flash e também remanejar as linhas de condensado provenientes do condicionador. Para essa instalação foi necessária a compra de alguns materiais tais como flanges, curvas, tubulações para condução do condensado, o tanque de flash, válvulas, purgadores, entre outros.

\subsection{REUTILIZAÇÃO DO CONDENSADO}

$\mathrm{O}$ vapor que troca calor indiretamente com a soja no condicionador, se condensa. Este condensado formado está a aproximadamente 10kgf/ $\mathrm{cm}^{2}$ (pressão com que o vapor vem da caldeira) e pode ser reaproveitado utilizando-se um tanque de flash à pressão de $1,1 \mathrm{kgf} / \mathrm{cm}^{2}$. Ao passar pelo tanque, há a formação de vapor flash e, neste caso, o vapor formado está sendo reaproveitado no expander. $\mathrm{O}$ condensado não aproveitado no tanque de flash retorna para a caldeira.

O expander, antes e depois das modificaçôes, está representado na figura abaixo.
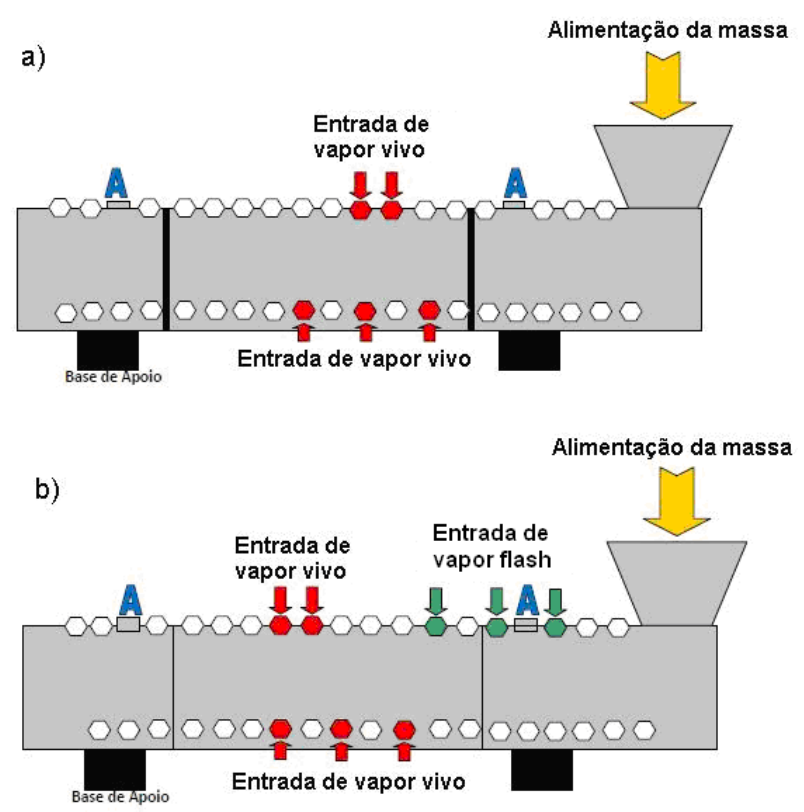

Figura 5: a) expander antes das modificaçōes, b) expander depois das modificaçóes.

Já o layout do setor antes e depois das modificações estão representados nas figuras 6 e 7 respectivamente (anexo).

\subsection{TESTE DE CONSUMO DE VAPOR}

O teste foi realizado na fábrica de óleos de uma cooperativa agroindustrial. Durante alguns dias mediu-se o consumo de vapor vindo da caldeira utilizando-se o tanque de flash e durante os dias restantes, fechou-se a linha do tanque flash, retornando o condensado diretamente para a caldeira e mediu-se o consumo de vapor e, entáo se mediu o consumo sem utilização deste tanque. $\mathrm{O}$ consumo foi medido utilizando-se um medidor Vortex, este medidor pode ser visualizado na figura abaixo.

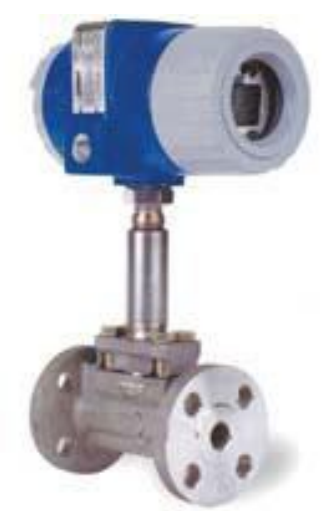

Figura 8: Medidor de vazão do tipo Vortex. 
Basicamente, de hora em hora, anotou-se a vazáo de vapor proveniente da caldeira e a vazão mássica de soja processada, visando-se encontrar o consumo médio de vapor por tonelada de soja processada.

Ao final do período de realizaçáo do teste, resumiu-se e tabelou-se os dados obtidos, sendo possível quantificar a economia de vapor devido à implantação do sistema flash. A tabela completa dos dados está no final deste trabalho (anexo).

Cabe ressaltar que após as mudanças no processo, esperaram-se alguns dias para que a fábrica voltasse a operar em regime permanente.

\subsection{METODOLOGIA DE CÁLCULO}

Com os dados de produçáo de soja e a quantidade de vapor utilizado, calculou-se o parâmetro V (denominado consumo específico e dado em quilos de vapor utilizado por tonelada de soja processada) através da seguinte equação:

$$
V=\frac{C}{P}
$$

Para obtenção da redução do consumo de vapor, calculou-se $\mathrm{V}_{\mathrm{SF}}$ e $\mathrm{V}_{\mathrm{CF}}$ A reduçáo é dada pela subtraçáo de $\mathrm{V}_{\mathrm{CF}}$ do valor obtido para $\mathrm{V}_{\mathrm{SF}}$ :

$$
R=V_{S F}-V_{C F}
$$

A economia ocasionada devido à redução de consumo de vapor pode ser encontrada através do seguinte cálculo:

$$
E=30 \cdot R \cdot P_{M} \cdot C_{T V}
$$

Para calcular o tempo de retorno do investimento realizado no processo de instalação do sistema flash:

$$
P B=\frac{I_{T}}{11 \cdot E}
$$

Onde, o numero 11 , do denominador, se refere aos meses de fabricação, no ano.

\section{CÁLCULOS E RESULTADOS}

Antes da instalação do sistema de flash o consumo específico médio era de:

$$
V_{S F}=316,86 \frac{\mathrm{kg}_{\text {VAPOR }}}{t_{\text {SOJA }}}
$$

Após a instalação do tanque o consumo específico médio caiu para:

$$
V_{C F}=300,52 \frac{\mathrm{kg}_{\text {VAPOR }}}{t_{\text {SOJA }}}
$$

Utilizando a equação (2):

$$
R=V_{S F}-V_{C F}=16,34 \frac{\mathrm{kg}_{\text {VAPOR }}}{t_{\text {SOJA }}}
$$

Com a produção média e com o preço de cada tonelada de vapor usado, pôde-se quantificar a economia alcançada devido à instalação do tanque de flash.

Seja o processamento médio de soja de 600 ton/dia e o preço de cada tonelada de vapor utilizado $\mathrm{R} \$ 27,19$. Portanto a economia no período de um mês foi de:

$$
\begin{gathered}
E=\left(16,34 \frac{\mathrm{kg}_{\text {VAPOR }}}{t_{\text {SOIA }}}\right) \cdot\left(600 \frac{t_{\text {SOIA }}}{\text { dia }}\right) \cdot 30 \frac{\text { dias }}{\text { mês }} \cdot 27,19 \frac{R \$}{t_{\text {VIAPOR }}} \cdot \frac{1 t}{1000 \mathrm{Kg}} \\
\mathrm{E}=7997,12 \mathrm{R} \$ / \mathrm{mês}
\end{gathered}
$$

$\mathrm{O}$ investimento total girou em torno de $\mathrm{R} \$$ $50.000,00$, portanto o payback do projeto é:

$$
\begin{gathered}
P B=\frac{50.000,00 R \$}{11 \frac{\text { meses }}{\text { ano }} \cdot 7997,12 \frac{R \$}{\text { mês }}} \\
P B=0,568 \text { ano } \\
P B \cong 6 \text { meses e } 24 \text { dias }
\end{gathered}
$$

\section{CONCLUSÃO}

Portanto, através dos dados obtidos no teste realizado, pôde-se concluir que a instalação do tanque flash acarretou uma redução de aproximadamente 5,16\% no consumo específico de vapor. Essa redução foi quantificada financeiramente, chegando a cerca de $\mathrm{R} \$ 8000,00$ mensais ( $\mathrm{R} \$$ 
$7997,12)$ e o payback calculado, relativamente baixo, mostrou que as mudanças propostas começarão a dar lucro para a empresa em pouco mais de 6 meses.

Ainda, observou-se que após implementadas as mudanças, os parâmetros operacionais, tais como temperatura do expander, pressão, umidade da massa, corrente do motor do expander, teor de óleo na massa que deixa o extrator, entre outros, não sofreram alteração.

Em vista dos resultados obtidos, a implementação do tanque de flash foi vantajosa, pois serviu para aproveitar uma energia que estava sendo perdida, acarretando ainda uma ótima redução de gastos com vapor.

\section{NOMENCLATURA}

\begin{tabular}{|c|c|}
\hline V & Consumo específico de vapor $\left(\mathrm{kg}_{\text {vapor }} / \mathrm{t}_{\text {soja }}\right)$ \\
\hline $\mathrm{C}$ & Média do consumo de vapor $(\mathrm{kg} / \mathrm{h})$ \\
\hline $\mathrm{P}$ & Quantidade média de soja processada $(\mathrm{t} / \mathrm{h})$ \\
\hline $\mathrm{R}$ & Redução no consumo de vapor $\left(\mathrm{kg}_{\text {vapor }} / \mathrm{t}_{\text {soja }}\right)$ \\
\hline $\mathrm{V}_{\mathrm{SF}}$ & $\begin{array}{l}\text { Consumo específico de vapor após a } \\
\text { instalação do tanque flash }\left(\mathrm{kg}_{\text {vapor }} / \mathrm{h}\right)\end{array}$ \\
\hline $\mathrm{V}_{\mathrm{CF}}$ & $\begin{array}{l}\text { Consumo específico de vapor antes da } \\
\text { instalação do tanque flash }\left(\mathrm{kg}_{\text {vapor }} / \mathrm{h}\right)\end{array}$ \\
\hline $\mathrm{E}$ & $\begin{array}{l}\text { Economia mensal devido à reduçáo no } \\
\text { consumo de vapor (R } \$ / \text { mês })\end{array}$ \\
\hline $\mathrm{P}_{\mathrm{M}}$ & $\begin{array}{l}\text { Média mensal do processamento de soja } \\
\qquad\left(\mathrm{t}_{\text {soja }}\right)\end{array}$ \\
\hline & Custo da tonelada de vapor \\
\hline PB & Payback \\
\hline $\mathrm{I}_{\mathrm{T}}$ & Investimento total $(\mathrm{R} \$)$ \\
\hline
\end{tabular}

\section{REFERÊNCIAS}

ABIOVE - Associação Brasileira das Indústrias de Óleos Vegetais.

Amaral, L.; Jaigobind, S. J.; Jaigobind, A. G. A. (2006). Óleo de Soja, Dossiê Técnico, Paraná; Instituto de Tecnologia do Paraná.
Barbosa, M. Z., de Freitas, S. M. e Franca, T. J. F. (1998). Consideraçóes sobre os desafios da cadeia de produção de óleo de soja no Brasil, Óleos \& Grãos, p. 44, Setembro/Outubro.

Bizzo, W. A. Geração, Distribuição e Utilização de Vapor. Universidade Estadual de Campinas, Faculdade de Engenharia Mecânica. Disponível em: <http://www.fem.unicamp.br/-em672>. Acesso em: 18/06/11.

Custódio, A. F. (2003). Modelagem e Simulação do Processo de Separação de óleo de Soja-Hexano por Evaporação. Dissertaçáo de Mestrado, Universidade de Campinas, Campinas.

Delmée, G. J. (2003). Manual de Medição de Vazão, $3^{a}$ Edição.

Erickson, D. R. (1995). Practical Handbook of Soybean Processing and Utilization, AOCS American Oils Chemists Society, Press and the United Soybean Board.

Lopes, K. S. (2008). Avaliação da Etapa de Clarificação do Óleo de Soja Através de Planejamento Composto Central e Investigação do Potencial de Melhoria Energética no Processamento da Soja. Dissertaçáo de Mestrado, Universidade Federal do Paraná, Curitiba.

Paraíso, P. R. (2001). Modelagem e análise do processo de obtençáo do óleo de soja. Tese de Doutorado, Universidade Estadual de Campinas, Campinas.

Passolongo, R., Ameida, E. M., Ramos, R. A. V. (2008). Proposta de Solução Para a Perda de Energia por "Vapor Flash" do Condensado de Escape de uma Usina Sucroalcooleira.

Savasini, J. A. A.(1981). Industrialização da Soja. In: Miyasaka,; ed. A Soja no Brasil, Campinas.

Thomas, G. C. (2003). Análise Teórico-Experimental da Extração de Óleo de Soja em Instalação Industrial do Tipo Rotocell. Tese de Doutorado, Porto Alegre. 
Tabela 1 - Consumo de vapor obtido através do Vortex.

\begin{tabular}{|c|c|c|c|c|}
\hline \multirow{2}{*}{ Análise } & \multicolumn{4}{|c|}{ Consumo de vapor } \\
\cline { 2 - 5 } & $\begin{array}{c}\text { Produção de soja } \\
(\mathrm{t} / \mathrm{h})\end{array}$ & $\begin{array}{c}\text { Consumo de vapor } \\
(\mathrm{t} / \mathrm{h})\end{array}$ & $\begin{array}{c}\text { Produção de soja } \\
(\mathrm{t} / \mathrm{h})\end{array}$ & $\begin{array}{c}\text { Consumo de vapor } \\
(\mathrm{t} / \mathrm{h})\end{array}$ \\
\hline 1 & 20,263 & 6,06 & 25,034 & 7,26 \\
\hline 2 & 25,040 & 7,37 & 25,403 & 7,94 \\
\hline 3 & 25,470 & 7,15 & 24,504 & 7,24 \\
\hline 4 & 24,986 & 7,77 & 25,268 & 7,51 \\
\hline 5 & 25,030 & 7,70 & 25,088 & 8,02 \\
\hline 6 & 24,905 & 7,25 & 24,625 & 8,07 \\
\hline 7 & 24,990 & 7,44 & 23,857 & 8,04 \\
\hline 8 & 25,109 & 7,45 & 24,641 & 8,17 \\
\hline 9 & 24,406 & 7,29 & 24,638 & 8,04 \\
\hline 10 & 22,382 & 7,44 & 24,372 & 8,13 \\
\hline Média & 24,258 & 7,29 & 24,743 & 7,84 \\
\hline
\end{tabular}
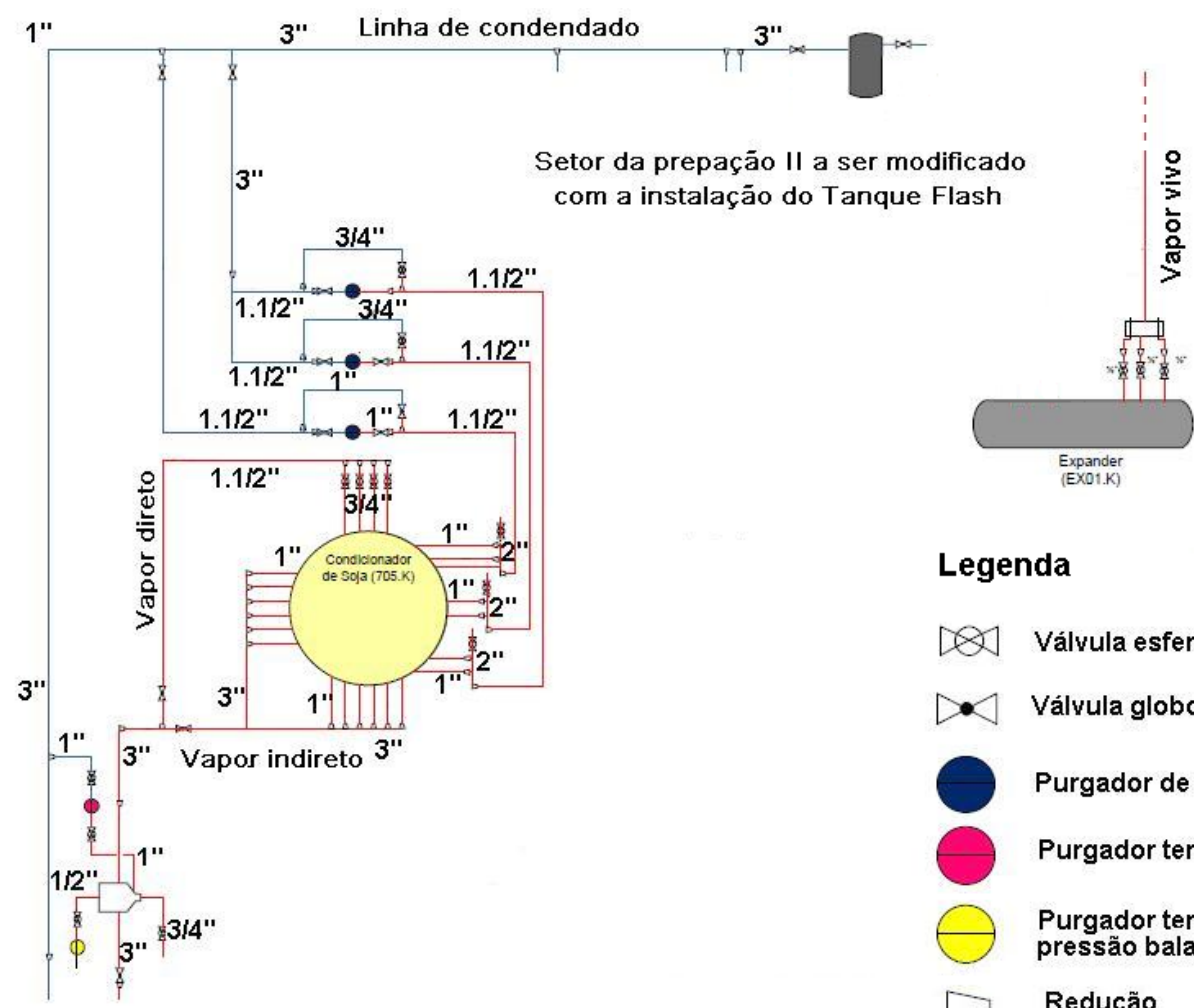

\section{Legenda}

$£$ Válvula esfera

. Válvula globo

Purgador de bóia

Purgador termodinâmico

Purgador termodinâmico de pressão balanceada

Redução

Figura 6: Setor da prepação antes da modificação proposta. 


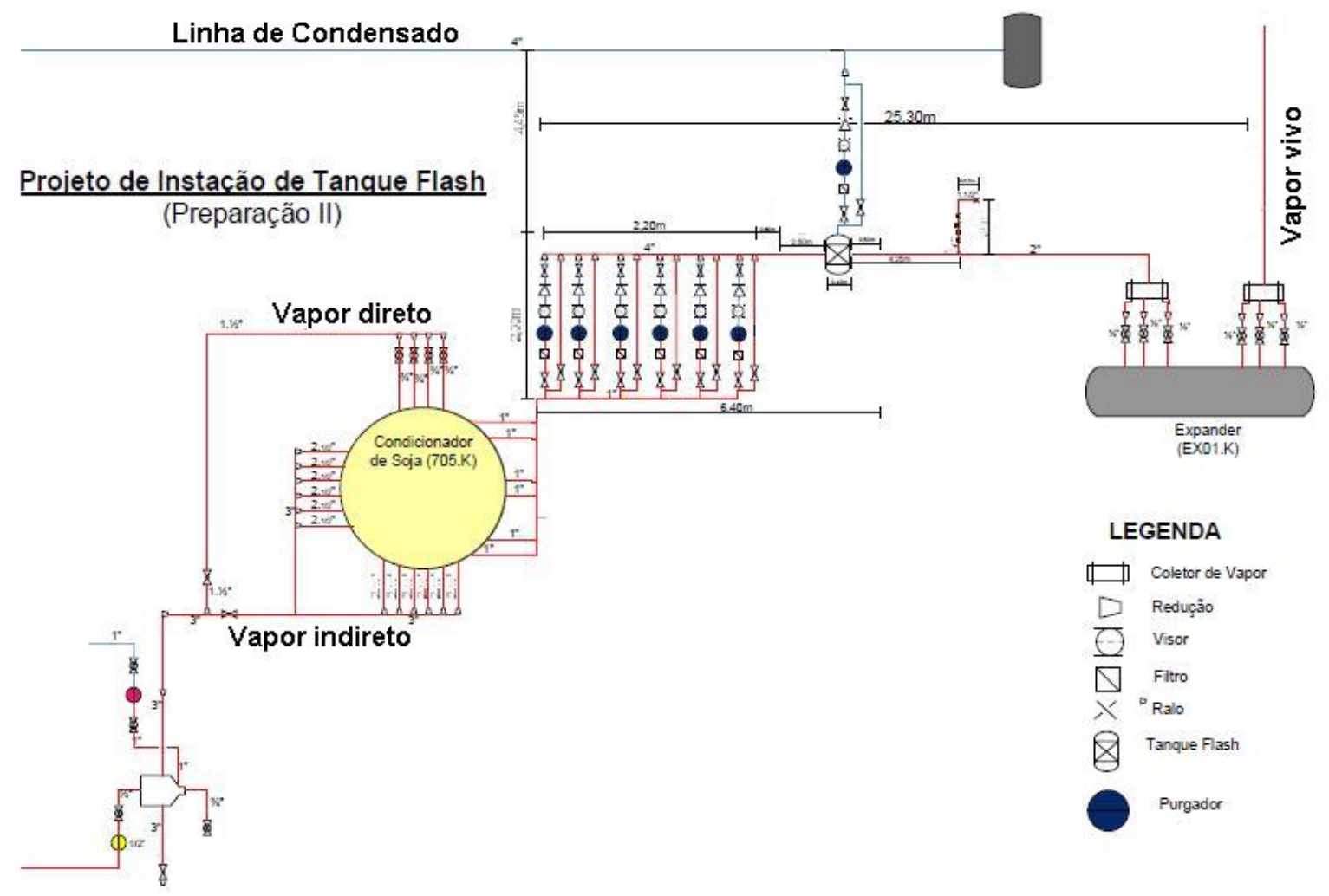

Figura 7: Setor da prepação após as modificações propostas. 